\title{
VARIACIÓN INTRANUAL EN EL ESPECTRO POLÍNICO DE LA MIEL PRODUCIDA EN HUARÁN (CUSCO, PERÚ)
}

\section{INTRAANNUAL VARIATION IN THE POLLEN SPECTRUM OF HONEY PRODUCEN IN HUARAN (CUSCO, PERÚ)}

\author{
Raúl Yuca-Rivas ${ }^{1}$
}

\begin{abstract}
Resumen
Con el fin de determinar cómo varía el espectro polínico de la miel, obtenida en colmenares ubicados en la localidad de Huarán, Cusco, durante la temporada apícola 2012 - 2013, e identificar las fuentes de polen y néctar usadas por Apis mellifera L., se analizaron en total 75 muestras de miel procedentes de 15 colmenas, en las que se realizaron cosechas sucesivas correspondientes a 5 periodos bimestrales de la temporada apícola: periodo I (julio - agosto 2012), periodo II (setiembre - octubre 2012), periodo III (noviembre - diciembre 2012), periodo IV (enero - febrero 2013) y periodo V (marzo - abril 2013); en todos los casos se realizaron preparaciones microscópicas acetolizadas. Los morfotipos polínicos obtenidos fueron reconocidos por comparación con una palinoteca de referencia. El número total de morfotipos polínicos determinados fue de 113: 40 en el periodo I, 55 en el periodo II, 77 en el periodo III, 75 en el periodo IV y 81 en el periodo V; las familias Asteraceae, Fabaceae y Lamiaceae aportaron mayor número de morfotipos. A través de un Análisis de Correspondencia, se evidenció una clara relación entre las especies visitadas por la abeja melífera y los periodos de producción, siendo las principales fuentes de polen y néctar Escallonia resinosa (Ruiz \& Pav.) Pers., y Eucalyptus sp. La composición del polen secundario y de menor importancia, fue similar durante toda la temporada. Se destacan Brassicaceae, Prunus sp. y Trifolium sp., en concordancia a sus amplios periodos de floración.
\end{abstract}

Palabras clave: melisopalinología, plantas melíferas, morfotipo polínico, Apis mellifera L., Huarán.

\begin{abstract}
In order to determine how the pollen spectrum changes in honey production, obtained from apiaries placed in Huarán, Cusco, during the beekeeping season 2012 - 2013 and to identify sources of pollen and nectar used by Apis mellifera L., 75 honey samples were analyzed from 15 hives obtained by successive harvests corresponding to 5 bimonthly periods of the beekeeping season: period I (July - August 2012), period II (September - October 2012), period III (November - December 2012), period IV (January - February 2013) and period V (March - April 2013); in all cases acetolized microscopic preparations were carried out. Pollen types obtained were identified by comparison with a reference collection. A total of 113 pollen types were identified: 40 in period I, 55 in period II, 77 in period III, 75 in period IV and 81 in period V; Asteraceae, Fabaceae and Lamiaceae were the families which contributed to the largest number of pollen types. By applying a correspondence analysis, a clear relationship between species visited by honeybees and production periods could be determined; Escallonia resinosa (Ruiz \& Pav.) Pers. and Eucalyptus sp. were main sources of pollen and nectar. The composition of secondary and minor pollen was similar over the whole season, with Brassicaceae, Prunus sp. and Trifolium sp. as the most important taxa, due to their long flowering periods.
\end{abstract}

Key words: melissopalynology, bee-plants, pollen types, Apis mellifera L., Huarán.

\section{Introducción.}

La miel es un agregado biológico muy complejo, cuya composición en polen y esencias, varía mucho en función de la flora visitada por Apis mellifera L., y de las condiciones climáticas y edafológicas del lugar donde se encuentran las colmenas (Martínez, 2010). La elección de las flores por la abeja melífera depende de muchos factores, pero críticamente y en primer lugar de la disponibilidad floral, es decir, depende de las especies vegetales cuya floración coincide con el 
momento del pecoreo, así como de la cantidad de flores disponibles (Sánchez, 2003; Martínez, 2010).

El análisis melisopalinológico, que consiste en el examen cualitativo y cuantitativo del contenido en granos de polen de una miel en estudio (Louveaux et al., 1978; Montenegro et al., 2003), ha sido usado para identificar plantas melíferas (fuentes de néctar y polen) usadas por la abeja melífera en una determinada región para la elaboración de miel, y de esta manera clasificarla de acuerdo a su origen botánico y geográfico (Louveaux et al., 1978).

Estudios melisopalinológicos para identificar la flora melífera, se han realizado en diversos países latinoamericanos como Brasil (Da Luz et al., 2007; Da Luz et al., 2010; Sereia et al., 2011), Chile (Montenegro et al., 2003; Montenegro et al., 2010), Colombia (Ortiz \& Cogua, 1989; Montoya, 2011; Nates-Parra et al., 2013; Montoya-Pfeiffer et al., 2014) y Argentina (Andrada \& Tellería, 2002; Forcone et al., 2003; Tellería et al. 2006; Caccavari \& Fagúndez, 2010). En el Perú se han realizado diversos estudios sobre determinación de flora melífera, potencial apícola y análisis sobre el origen botánico de mieles (León et al., 1989-1990; Bonino \& Paucarmayta, 2002).

El objetivo principal de este estudio, fue determinar la variación intranual en el espectro polínico de la miel producida en la localidad de Huarán (Valle Sagrado de los Incas, Cusco), mediante el estudio de mieles provenientes de cosechas sucesivas o escalonadas, y así poder detectar las fuentes de polen y néctar usadas por la abeja melífera durante toda una temporada apícola, lo que pueda justificar la cosecha por etapas y plantear estrategias de conservación para la flora melífera, ya que muchas son especies nativas (Bonino \& Paucarmayta, 2002).

\section{Materiales y métodos.}

Características del área de estudio

La localidad de Huarán está ubicada a 59 kilómetros de la ciudad del Cusco, en el Valle Sagrado de los Incas, correspondiente a la provincia Calca y región Cusco, entre las coordenadas UTM 18S 0821575E $8527152 \mathrm{~N}$ y $0820635 \mathrm{E} 8525713 \mathrm{~N}$ y con altitudes que oscilan entre los 2800 y 3000 msnm (Figura 1). La zona presenta, en general, un clima templado-cálido subhúmedo. La precipitación total anual varía entre 460 y $550 \mathrm{~mm}$. De acuerdo al Mapa Ecológico del Perú, esta zona corresponde al bosque seco Montano Bajo Subtropical (bs-MBS) (Pérez, 2009).

\section{Procedimiento de muestreo}

Los apiarios de Huarán se seleccionaron por tener una producción representativa de miel en el Valle Sagrado de los Incas, con 30 colmenas en promedio por cada uno de sus 5 apiarios. Para la selección del conjunto de réplicas o muestra, se procedió con un muestreo aleatorio, seleccionando 15 colmenas distribuidas por todos los apiarios; todas las colmenas fueron de tipo Langstroth. Con respecto a las abejas, los enjambres pertenecían a la especie Apis mellifera $\mathrm{L}$.

Para su determinación botánica se colectaron especies vegetales en floración ubicadas en un área de $700 \mathrm{~m}$ de radio alrededor de los apiarios; adicionalmente, se colectaron flores en antesis de cada planta, estas sirvieron para la elaboración de una palinoteca de referencia. La determinación de las especies fue un trabajo realizado en el Herbario Vargas CUZ de la Facultad de Ciencias de la Universidad Nacional de San Antonio Abad del Cusco (UNSAAC).

Las mieles estudiadas correspondieron a la temporada apícola 2012 - 2013, en la que se definieron cinco periodos bimestrales de producción: periodo I (julio-agosto 2012), periodo II (setiembreoctubre 2012), periodo III (noviembre-diciembre 2012), periodo IV (enero-febrero 2013) y periodo V (marzo-abril 2013). Se colocaron en cada una de las colmenas seleccionadas, entre 1 y 5 marcos de madera debidamente rotulados al inicio de cada periodo de producción, estos fueron extraídos y reemplazados al final de dichos periodos; se procuró que por lo menos unas $3 / 4$ partes del panal construido estuviera

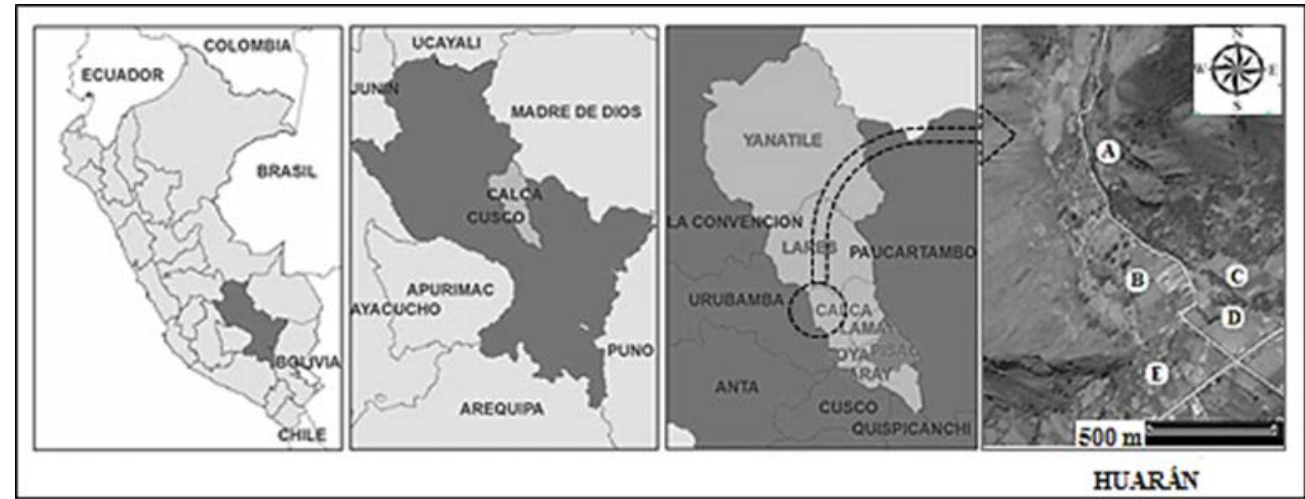

Figura 1. Mapa de ubicación geográfica y política de la localidad de Huarán. A, B, C, D y E: ubicación de los apiarios. 
operculado. La miel se extrajo por prensado de los panales, excluyendo el polen que pudiera haber estado almacenado en las celdas. Una vez extraída la miel, se procedió a su homogenización, de la cual se separó 50 g por colmena, obteniéndose 15 mieles por periodo de producción siendo en total 75 para toda la temporada apícola.

\section{Análisis palinológico}

Las anteras de las flores se procesaron por el método de acetólisis (Erdtman, 1960), posteriormente se hizo el análisis microscópico, obteniéndose los morfotipos polínicos de referencia de la palinoteca deposita en el Herbario Vargas CUZ.

Se prepararon 2 láminas microscópicas acetolizadas por miel; para ello se siguió la metodología propuesta por Louveaux et al. (1978), con algunas modificaciones sugeridas por Von der Ohe et al. (2004).

Las observaciones al Microscopio Electrónico de Barrido (MEB), fueron realizadas con un equipo Philips - SEM 505 y un digitalizador de imagen Soft Imaging Sistem ADDA II, sobre el sedimento polínico acetolizado (Figura 2).

El análisis cualitativo por miel, fue realizado mediante el recuento de 1200 granos de polen sobre las 2 preparaciones microscópicas. La determinación de los morfotipos polínicos se realizó al nivel de especie cuando ha sido posible, en otros casos se llegó a género, tribu o familia. Para la determinación se recurrió a la palinoteca de referencia preparada previamente $\mathrm{y}$ al uso de numerosas publicaciones especializadas (Sáenz, 1978; Carretero, 1989; Colinvaux et al., 1999; Andrada \& Tellería, 2005; Pardillo \& La-Serna, 2007; Hesse et al., 2009; Barth
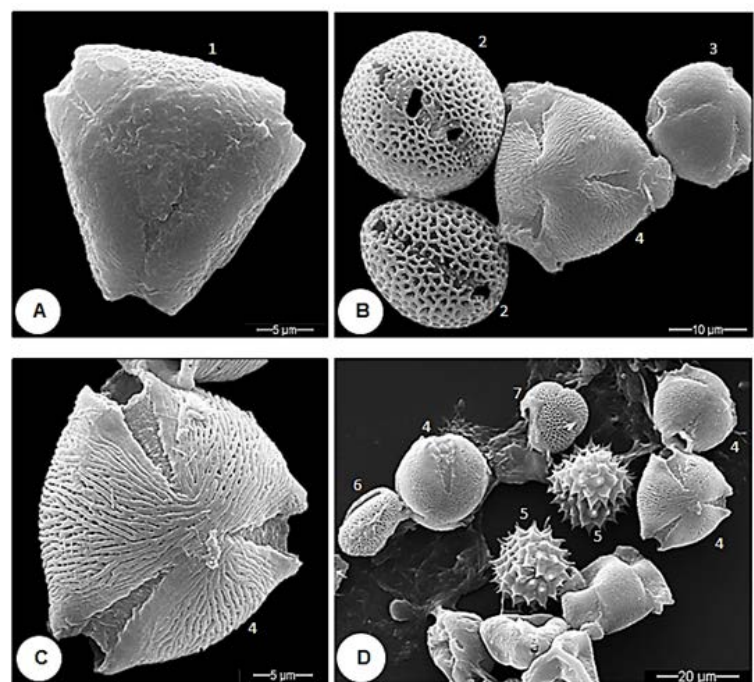

Figura 2. Microfotografías electrónicas de barrido de los principales morfotipos polínicos: Eucalyptus sp. (1-A), Brassica sp. (2-B), Escallonia resinosa (3-B), Prunus serotina (4-B, C y D), Asteraceae morfotipo 2 (5-D), Salix sp. (6D), Brassicaceae (7-D). et al., 2012).

Los morfotipos polínicos, de acuerdo a sus porcentajes (espectro), fueron clasificados en: $>45 \%$, polen dominante (D); $16-45 \%$, polen secundario o acompañante (S); 3-16 \%, polen de menor importancia (I); $1-3 \%$, polen en trazas o raro $(\mathrm{T}) ;<1 \%$, Polen esporádico o presente $(+)$. Las mieles en las cuales un morfotipo polínico estuvo representado en $\geq 45 \%$, fueron clasificadas como monoflorales, y aquellas en las que no se superó este porcentaje, como multiflorales o mixtas (Louveaux et al., 1978).

Análisis estadístico

Con la finalidad de observar la relación entre el espectro polínico de las mieles y los periodos de producción, se aplicó un Análisis de Correspondencia, para ello se utilizó el software "Statistica” versión $7{ }^{\circledR}$. En este análisis, se consideraron todos los morfotipos polínicos presentes en el espectro de las mieles.

\section{Resultados.}

Se determinó un total de 113 morfotipos polínicos en todas las mieles analizadas, 40 en el periodo I, 55 en el periodo II, 77 en el periodo III, 75 en el periodo IV y 81 en el periodo V; siendo Asteraceae, Fabaceae y Lamiaceae las familias que aportaron mayor número de morfotipos.

Los morfotipos polínicos fueron determinados a diferentes niveles taxonómicos, 41 a nivel de especie, 45 a género, 13 a tribu y 14 a familia. La Tabla 1 muestra dichos morfotipos agrupados por familias, con sus correspondientes frecuencias de ocurrencia (F.O.) en el total de las mieles analizadas, y en cada una de las clases de frecuencia para los cinco de periodos de producción considerados.

El 58 \% de los morfotipos polínicos pertenecieron a plantas nativas, como en el caso de Aegiphila mortonii Moldenke, Escallonia resinosa (Ruiz \& Pav.) Pers., Gaiadendron punctatum (Ruiz \& Pav.) G. Don, Pineda incana Ruiz \& Pav., y Weinmannia producta Moricand. El estrato mejor representado fue el herbáceo (48.67\%), seguido del arbustivo (28.32 \%) y el arbóreo (22.12 \%), mientras que el epifito solo en $0.88 \%$.

Durante el quinto periodo de producción el morfotipo polínico de Escallonia resinosa (Ruiz \& Pav.) Pers., se presentó como dominante en tres de las mieles analizadas, en el primer y cuarto periodos como secundario y en el tercero como de menor de importancia, en dichos periodos su frecuencia de ocurrencia fue del 100 \%. Eucalyptus sp. se presentó como polen secundario del primer al cuarto periodos, $\mathrm{y}$ en el quinto como polen de menor importancia, su frecuencia de ocurrencia también fue del $100 \%$. Otras taxa que destacaron durante toda la temporada apícola fueron Brassicaceae, Prunus sp., Telima monspessulanus L. y Trifolium sp., en concordancia a sus amplios y abundantes periodos de floración. 
El polen de plantas poliníferas fue detectado durante todos los periodos de producción con frecuencias relativas menores al $1 \%$, con excepción del polen de Alnus acuminata Kunth, Vallea stipularis L. f. y Morella pubescens (Humb. \& Bonpl. Ex Willd) Wilbur, que aparecieron con porcentajes entre 1 y $3 \%$ en algunas de las mieles.

El Análisis de Correspondencia permitió determinar la relación entre los morfotipos polínicos y los periodos de producción. La Figura 3, muestra a los morfotipos polínicos que más influyen en la asociación. Los valores singulares, el porcentaje de inercia, el porcentaje acumulado y los valores ChiCuadrado, se muestran en la Tabla 2. El porcentaje acumulado de los dos primeros ejes explica el $86.57 \%$ de la variación.

\section{Discusión.}

El análisis melisopalinológico de la miel, se basa en el supuesto de que la abeja melífera, al recoger el néctar de una flor, arrastra también una determinada cantidad de polen de esa especie en dicho néctar, la cual permanece en la miel y permite conocer su origen floral (García, 2003).

Asteraceae, Fabaceae y Lamiaceae estuvieron mejor representadas en los espectros polínicos, debido a su abundancia en la flora local. Por otra parte, su morfología floral y oferta de néctar se prestan adecuadas al trabajo de pecoreo de las obreras, como observaron Andrada \& Tellería (2002), Bonino \& Paucarmayta (2002), Forcone et al. (2003) y Caccavari \& Fagúndez (2010). Asteraceae aportó la mayor cantidad de morfotipos polínicos durante la estación lluviosa (diciembre - abril) y Fabaceae durante la estación de secas (julio - noviembre).

A través del análisis estadístico, se pudo evidenciar que las variaciones en la floración a lo largo del año, afectan de manera relevante el contenido polínico de las mieles producidas, siendo las principales fuentes de polen y néctar Escallonia resinosa (Ruiz \& Pav.) Pers., y Eucalyptus sp. Al comparar los espectros polínicos de los 5 periodos de producción, se descubrieron variaciones importantes, tanto referido a la identidad de los morfotipos como a sus abundancias relativas, esto debido a muchos factores, como la estructura poblacional del recurso vegetal, la cantidad de flores producidas, la accesibilidad a los nectarios, la vistosidad de las flores o el incremento en la floración conforme se va pasando hacia la estación lluviosa.

Fue solo entre marzo y abril, que se registró la producción de mieles monoflorales de Escallonia resinosa (Ruiz \& Pav.) Pers., coincidiendo con el momento de máxima floración de dicha especie, la cual era bastante abundante en la localidad de estudio, estas podrían ser catalogadas como mieles de “chachacomo". La promoción de la certificación de miel monofloral de Escallonia, y de este modo de su origen, es altamente recomendable (Montenegro et al., 2010). Pero sería necesario evaluar también sus características organolépticas y físico-químicas (Accorti et al., 1986). Las mieles restantes fueron catalogadas como multiflorales o de mil flores, aunque se podría recomendar la catalogación de mieles "biflorales”, como sugieren Montenegro et al. (2010), de Eucalyptus sp. y Prunus sp., para los meses de setiembre y octubre.

La práctica actual en el área de estudio, corresponde a una sola cosecha anual al final de la temporada apícola, extrayéndose una mixtura de mieles provenientes de diferentes floraciones; a partir de lo encontrado en esta investigación, se podría sugerir dos cosechas anuales, una entre octubre y noviembre y la otra entre marzo y mayo. Se requieren

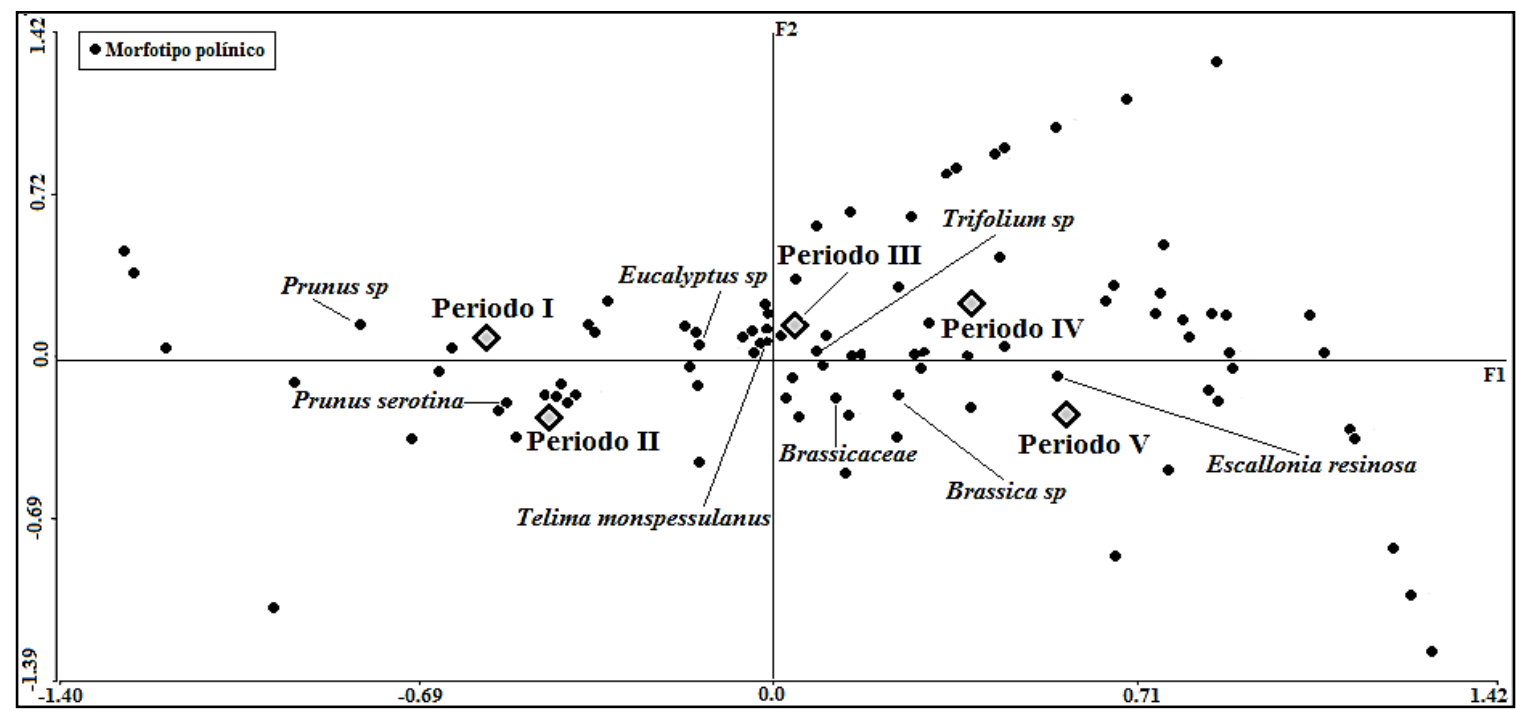

Figura 3. Diagrama de correspondencia para los principales morfotipos polínicos y su relación con los periodos de producción. 
más indagaciones que confirmen esta posibilidad.

Respecto a los granos de polen de plantas poliníferas (que no aportan néctar), se consideró que su presencia fue debido a que las obreras, cuando no recolectan néctar, transportan polen puro a manera de papilla en las corbículas de sus patas traseras y en sus cuerpos para el sustento de la colmena, y tras entrar en esta de una u otra manera llegan a “contaminar” la miel en proceso de elaboración, como así sucedió con el polen de Alnus acuminata Kunth (Sayas \& Huamán, 2009). Otra posible explicación a su presencia, está relacionada al transporte por el viento, que ocasionalmente lleva estos granos de polen a caer sobre el néctar de flores actinomorfas o directamente en la colmena.

\section{Conclusiones.}

Se determinaron 113 morfotipos polínicos en la miel de Huarán durante los cinco periodos bimestrales de producción correspondientes a la temporada apícola 2012-2013, siendo Asteraceae, Fabaceae y Lamiaceae las mejor representadas. Se encontraron variaciones importantes al comparar los espectros polínicos de los periodos de producción, resaltando los cambios acaecidos en Eucalyptus sp. y Escallonia resinosa (Ruiz \& Pav.) Pers., siendo esta última fuente de mieles monoflorales. Brassicaceae, Prunus sp., Telima monspessulanus L., y Trifolium sp., se presentaron constantes durante toda la temporada apícola.

\section{Agradecimientos.}

A los apicultores de Huarán, especialmente a Jerry y Jeremías por todas las facilidades prestadas. A la M. Sc. Fructuosa De La Torre por el acceso al Herbario Vargas CUZ, a la Quim. Alicia Claverí por el acceso al laboratorio de Bioquímica de la UNSAAC y finalmente al M. Sc. Wilfredo Chávez por sus aportes en el desarrollo del análisis estadístico.

\section{Literatura citada.}

Accorti M., Persano L., Piazza M. \& Sabatini A. 1986 Schede di caratterizzazione delle principali qualita di miele italiano. Apicoltura. 2: $1-35$.

Andrada A. C. \& Tellería M. C. 2002. Botanical origin of honey from south of Calden district (Argentina). Grana. 41: $58-62$.

2005. Pollen collected by honeybees (Apis mellifera L.) from south of Caldén district (Argentina): Botanical origin and protein content. Grana. 44: 1 - 8 .

Barth O. M., Freitas A. S. \& Almeida-Muradian L. 2012. Palynological analysis of Brazilian stingless bee pothoney. S.A.B.E.R.-U.L.A., Universidad de los Andes, Mérida, Venezuela, p. $1-8$.

Bonino R. E. \& Paucarmayta D. J. 2002. Evaluación del potencial de la flora melífera en la margen derecha del rio Mapacho, sector Challabamba - Acobamba. PROMANU, Cusco.
Caccavari M. \& Fagúndez G. 2010. Pollen spectra of honeys from the Middle Delta of Paraná River (Argentina) and their environmental relationship. Spanish Journal of Agricultural Research, 8 (1): 42 - 52.

Carretero J. L. 1989. Análisis polínico de la miel. Madrid: Ediciones Mundi - Prensa, España.

Colinvaux P., De Oliveira P. E. \& Moreno J. E. 1999. Amazon Pollen Manual and Atlas. Singapore: Overseas Publishers Association.

Da Luz C. F., Thomé M. L. \& Barth O. M. 2007. Recursos tróficos de Apis mellifera L. (Hymenoptera, Apidae) na região de Morro Azul do Tinguá, Estado do Rio de Janeiro. Rev. Bras. De Bot. 30: 29 - 36.

, Bacha G. L., Fonseca R. \& Sousa P. 2010. Comparative pollen preferences by africanized honeybees Apis mellifera L. of two colonies in Pará de Minas, Minas Gerais, Brazil. Anais da Academia Brasileira de Ciencias. 82 (2): 293 - 304.

Erdtman G. 1960. The acetolysis method, a revised description. Sven Bot Tidskr. 54: 561 - 564.

Forcone A., Bravo O. \& Ayestarán M. 2003. Intraannual variations in the pollinic spectrum of honey from the lower valley of the River Chubut (Patagonia, Argentina). Spanish Journal of Agricultural Research. 1 (2): 29 - 36.

García R. 2003. Estudio palinológico y colorimétrico de mieles monoflorales de la región de Murcia. Cartagena: Escuela Técnica Superior de Ingeniería Agronómica, Universidad Politécnica de Cartagena.

Hesse M., Halbritter H., Zetter R., Weber M., Buchner R., Frosch - Radivo A. \& Ulrich S. 2009. Pollen Terminology: An illustrated handbook. Vienna: Springer Wien New York, Austria.

León O., Balbín J., Villa V. \& Isayama V. 1989 - 1990. Origen botánico de la miel del Valle del Mantaro. Zonas Áridas. 6: 95-108.

Louveaux J., Maurizio A. \& Vorwohl G. 1978. Methods of Melissopalynology. Bee World. 59: 139 - 157.

Martínez S. 2010. Caracterización melisopalinológica de las cargas de polen recolectadas por Apis mellifera L. 1758, y su relación con la estructura y composición florística local. Servicio de Medio Ambiente de la Diputación de Toledo.

Montenegro G., Pizarro R., Ávila G., Castro R., Ríos C., Muñoz O., Bas F. \& Gómez M. 2003. Origen botánico y propiedades químicas de las mieles de la región mediterránea árida de Chile. Ciencia e Investigación Agraria. 30: 161 - 174.

, Peña R. C. \& Pizarro R. 2010. Multivariate analysis of pollen frequency of the native species Escallonia pulverulenta (Saxifragaceae) in Chilean honeys. Revista Brasil. Bot. 4 (33): 615 - 630.

Montoya-Pfeiffer P., León-Bonilla D. \& Nates-Parra G. 2014. Catálogo de polen en mieles de Apis mellifera L., provenientes de zonas cafeteras en la Sierra Nevada de Santa Marta, Magdalena, Colombia. Rev. Acad. Colomb. Cienc. Ex. Fis. Nat. 38 (149): 364-384.

Montoya P. M. 2011. Uso de recursos florales poliníferos por Apis mellifera L. (Hymenoptera: Apidae) en apiarios de la Sabana de Bogotá y alrededores. Colombia. Tesis M. Sc. Universidad Nacional de Colombia.

Nates-Parra G., Montoya P., Chamorro F., Ramírez N., Giraldo C. \& Obregón D. 2013. Origen geográfico y botánico de mieles de Apis mellifera (Apidae) en cuatro 
departamentos de Colombia. Acta biol. Colomb. 18 (3): 427-438.

Ortiz D. \& Cogua J. 1989. Reconocimiento de granos de polen de algunas plantas melíferas en la sabana de Bogotá. Agronomía Colombiana. 6: 52 - 63.

Pardillo L. \& La Serna I. E. 2007. Espectro polínico de algunas mieles producidas en Tenerife y La Gomera (Islas Canarias, España). Geo - Eco - Trop. 31: 215 232.

Pérez I. M. 2009. Variabilidad morfológica de cultivares de Oxalis tuberosa Mol., en tres comunidades campesinas del distrito de Pisac. Tesis Blga. Universidad Nacional de San Antonio Abad del Cusco.

Sáenz C. 1978. Polen y Esporas: Introducción a la palinología y vocabulario palinológico. (Primera edición). Madrid: Ediciones H. Blume. España.
Sánchez C. 2003. Crianza y producción de abejas Apicultura. Lima: Ediciones Ripalme, Perú.

Sayas R. \& Huamán L. 2009. Determinación de la flora polinífera del valle de Oxapampa (Pasco, Perú) en base a estudios palinológicos. Ecología Aplicada. 8 (2): 53 59.

Sereia M. J., Alves E. M., Toledo V. A., Marchini L. M., Sekine E. S., Faquinello P., Almeida D. \& Moreti A. C. 2011. Physicochemical characteristics and pollen spectra of organic and non-organic honey samples of Apis mellifera L. An. Acad. Bras. Cienc. 83 (3): 1077 - 1090.

Tellería M., Salgado C. \& Andrada A. 2006. Rhamnaceae asociadas a mieles fétidas en Argentina. Rev. Mus. Argentino Cienc. Nat. 8 (2): 237 - 241.

Von Der Ohe W., Persano L., Piana M. L., Morlot M. \& Martin P. 2004. Harmonized methods of Melissopalynology. Apidologie. 35: S18 - S35. 
Tabla 1. Clases de frecuencia y frecuencia de ocurrencia de los 113 morfotipos polínicos determinados en los 5 periodos de producción en la localidad de Huarán para la temporada apícola 2012-2013.

\begin{tabular}{|c|c|c|c|c|c|c|c|c|c|c|c|c|c|c|c|c|c|c|c|c|c|c|c|c|c|c|c|}
\hline \multirow{2}{*}{ FAMILIA } & \multirow{2}{*}{ MORFOTIPO POLÍNICO } & \multicolumn{5}{|c|}{ Periodo I } & \multicolumn{5}{|c|}{ Periodo II } & \multicolumn{5}{|c|}{ Periodo III } & \multicolumn{5}{|c|}{ Periodo IV } & \multicolumn{6}{|c|}{ Periodo V } \\
\hline & & $\mathrm{S}$ & I & $\mathrm{T}$ & $(+)$ & FO & $\mathrm{S}$ & $\mathrm{I}$ & $\mathrm{T}$ & $(+)$ & FO & $\mathrm{S}$ & $\mathrm{I}$ & $\mathrm{T}$ & $(+)$ & FO & $\mathrm{S}$ & I & $\mathrm{T}$ & $(+)$ & FO & $\mathrm{D}$ & $\mathrm{S}$ & $\mathrm{I}$ & $\mathrm{T}$ & $(+)$ & FO \\
\hline ADOXACEAE & Sambucus peruviana Kunth * & & & & & & & & & & & & & & 2 & 13 & & & & & & & & & & & \\
\hline \multirow[t]{2}{*}{ AMARANTHACEAE } & Amaranthaceae & & & & & & & & & & & & & & 4 & 27 & & & & 5 & 33 & & & & & & \\
\hline & Iresine $s p$ & & & & & & & & & & & & & & 2 & 13 & & & & 3 & 20 & & & & & & \\
\hline AMARYLLIDACEAE & Amaryllidaceae & & & 1 & 11 & 80 & & & & 2 & 13 & & & & & & & & & & & & & & & & \\
\hline ANACARDIACEAE & Schinus sp * & & 15 & & & 100 & & 15 & & & 100 & & 14 & 1 & & 100 & & 1 & 12 & & 87 & & & 6 & 9 & & 100 \\
\hline \multirow[t]{2}{*}{ APIACEAE } & Apiaceae & & & & & & & & & 4 & 27 & & & & 5 & 33 & & & & 5 & 33 & & & & & 4 & 27 \\
\hline & Conium maculatum L. & & & & 11 & 73 & & & & 8 & 53 & & & & 7 & 47 & & & & 8 & 53 & & & & & 6 & 40 \\
\hline \multirow[t]{26}{*}{ ASTERACEAE } & Ageratina $s p *$ & & & 15 & & 100 & & & 6 & 2 & 53 & & & 6 & 3 & 60 & & & 1 & 9 & 67 & & & & 4 & 5 & 60 \\
\hline & Anthemideae & & & 8 & 4 & 80 & & & & 8 & 53 & & & 1 & 7 & 53 & & & 2 & 7 & 60 & & & & & 7 & 47 \\
\hline & Asteraceae morfotipo 1 & & & & 5 & 33 & & & & 2 & 13 & & & & & & & & & & & & & & & & \\
\hline & Asteraceae morfotipo 2 & & & & & & & & & & & & & & 2 & 13 & & & & 3 & 20 & & & & & 2 & 13 \\
\hline & Asteraceae morfotipo 3 & & & & & & & & & & & & & & & & & & & & & & & & & 3 & 20 \\
\hline & Astereae morfotipo 1 & & & 12 & & 80 & & & 4 & 4 & 53 & & & & 10 & 67 & & & 1 & 9 & 67 & & & & & 9 & 60 \\
\hline & Astereae morfotipo 2 & & & & & & & & & & & & & & & & & & & & & & & & & 5 & 33 \\
\hline & Astereae morfotipo 3 & & & & & & & & & & & & & & & & & & & & & & & & & 2 & 13 \\
\hline & Baccharis sp * & & & & & & & 15 & & & 100 & & 14 & 1 & & 100 & & 6 & 6 & & 80 & & & 3 & 8 & & 73 \\
\hline & Barnadesia horrida Muschl.* & & & & 7 & 47 & & & & & & & & & & & & & & & & & & & & 3 & 20 \\
\hline & Bidens $s p *$ & & & 12 & 1 & 87 & & 1 & 14 & & 100 & & & 15 & & 100 & & 13 & 2 & & 100 & & & 1 & 12 & & 87 \\
\hline & Eupatorieae morfotipo 1 & & & & 6 & 40 & & & & & & & & & & & & & & & & & & & 3 & 5 & 53 \\
\hline & Eupatorieae morfotipo 2 & & & & & & & & & & & & & & 8 & 53 & & & & 9 & 60 & & & & 4 & 3 & 47 \\
\hline & Eupatorieae morfotipo 3 & & & & & & & & & & & & & & 3 & 20 & & & & 3 & 20 & & & & & 3 & 20 \\
\hline & Helianthus annuus L. & & & & & & & & & 5 & 33 & & & & 4 & 27 & & & & 4 & 27 & & & & & 3 & 20 \\
\hline & Heliantheae morfotipo 1 & & & & & & & & & & & & & & 7 & 47 & & & & 8 & 53 & & & & 4 & 4 & 53 \\
\hline & Heliantheae morfotipo 2 & & & & & & & & & & & & & & & & & & & & & & & & & 6 & 40 \\
\hline & Inuleae & & & & & & & & & & & & & & & & & & & & & & & & & 3 & 20 \\
\hline & Jungia sp * & & & & & & & & & & & & 2 & 12 & & 93 & & 13 & 2 & & 100 & & & & 15 & & 100 \\
\hline & Lactuceae morfotipo 1 & & & & 9 & 60 & & & & 9 & 60 & & & & 8 & 53 & & & & 10 & 67 & & & & & 7 & 47 \\
\hline & Lactuceae morfotipo 2 & & & & & & & & & & & & & & & & & & & & & & & & & 1 & 7 \\
\hline & Mutisieae * & & & & & & & & & & & & & & & & & & & & & & & & & 4 & 27 \\
\hline & Ophryosporus sp * & & & 6 & 3 & 60 & & & & & & & & & & & & & & & & & & & & & \\
\hline & Senecio $s p *$ & & & & & & & & & 2 & 13 & & & & & & & & & & & & & & & & \\
\hline & Tagetes $s p *$ & & & & 8 & 53 & & & & & & & & & & & & & & & & & & & & 7 & 47 \\
\hline & Verbesina auriculigera S. F. Blake * & & & 11 & 1 & 80 & & 14 & 1 & & 100 & & 15 & & & 100 & & 15 & & & 100 & & & 13 & 2 & & 100 \\
\hline BEGONIACEAE & Begonia sp & & & & & & & & & 4 & 27 & & & & 4 & 27 & & & & 3 & 20 & & & & & 3 & 20 \\
\hline BERBERIDACEAE & Berberis $s p *$ & & & & & & & & & & & & & & 8 & 53 & & & & 3 & 20 & & & & & & \\
\hline BETULACEAE & Alnus acuminata Kunth * & & & & & & & & & 15 & 100 & & & 14 & 1 & 100 & & & 15 & & 100 & & & & 1 & 12 & 87 \\
\hline BIGNONIACEAE & Tecoma stans (Kunth) J.R.I. Wood * & & & & 7 & 47 & & & & 7 & 47 & & & & 7 & 47 & & & & & & & & & & 7 & 47 \\
\hline
\end{tabular}


Tabla 1. Clases de frecuencia y frecuencia de ocurrencia de los 113 morfotipos polínicos determinados en los 5 periodos de producción en la localidad de Huarán para la temporada apícola 2012-2013.

\begin{tabular}{|c|c|c|c|c|c|c|c|c|c|c|c|c|c|c|c|c|c|c|c|c|c|c|c|c|c|c|c|}
\hline \multirow{2}{*}{ FAMILIA } & \multirow{2}{*}{ MORFOTIPO POLÍNICO } & \multicolumn{5}{|c|}{ Periodo I } & \multicolumn{5}{|c|}{ Periodo II } & \multicolumn{5}{|c|}{ Periodo III } & \multicolumn{5}{|c|}{ Periodo IV } & \multicolumn{6}{|c|}{ Periodo V } \\
\hline & & $\mathrm{S}$ & $\mathrm{I}$ & $\mathrm{T}$ & $(+)$ & FO & $\mathrm{S}$ & I & $\mathrm{T}$ & $(+)$ & FO & $\mathrm{S}$ & I & $\mathrm{T}$ & $(+)$ & FO & $\mathrm{S}$ & I & $\mathrm{T}$ & $(+)$ & FO & $\mathrm{D}$ & $\mathrm{S}$ & $\mathrm{I}$ & $\mathrm{T}$ & $(+)$ & FO \\
\hline \multirow[t]{2}{*}{ BORAGINACEAE } & Boraginaceae & & & & & & & & & & & & & & & & & & & & & & & & & 2 & 13 \\
\hline & Phacelia pinnatifida Weddell * & & & & & & & & & & & & & & 2 & 13 & & & & 3 & 20 & & & & & 3 & 20 \\
\hline \multirow[t]{2}{*}{ BRASSICACEAE } & Brassica sp & & & 12 & 3 & 100 & & 15 & & & 100 & & 15 & & & 100 & & 11 & 4 & & 100 & & & 15 & & & 100 \\
\hline & Brassicaceae & & 6 & 9 & & 100 & & 15 & & & 100 & & 15 & & & 100 & & 15 & & & 100 & & & 15 & & & 100 \\
\hline BROMELIACEAE & Bromeliaceae & & & & & & & & & & & & & & & & & & & & & & & & & 2 & 13 \\
\hline CACTACEAE & Opuntia ficus-indica (L.) Miller & & & & & & & & & 3 & 20 & & & & 3 & 20 & & & & & & & & & & & \\
\hline \multirow{2}{*}{ CARYOPHYLLACEAE } & Caryophyllaceae morfotipo 1 & & & & & & & & & & & & & & & & & & & 3 & 20 & & & & & 2 & 13 \\
\hline & Caryophyllaceae morfotipo 2 & & & & & & & & & & & & & & & & & & & & & & & & & 3 & 20 \\
\hline CONVOLVULACEAE & Convolvulus $s p *$ & & & & & & & & & 4 & 27 & & & & 5 & 33 & & & & 6 & 40 & & & & & & \\
\hline \multirow[t]{3}{*}{ CUCURBITACEAE } & Cucurbita sp * & & & & & & & & & & & & & & 3 & 20 & & & & 2 & 13 & & & & & 2 & 13 \\
\hline & Cyclanthera $s p *$ & & & & & & & & & & & & & & & & & & & & & & & & & 4 & 27 \\
\hline & Sicyos baderoa Hooker \& Arnott* & & & & & & & & & & & & & & & & & & & & & & & & & 8 & 53 \\
\hline CUNONIACEAE & Weinmannia producta Moricand * & & & 5 & 4 & 60 & & & 8 & 3 & 73 & & & 10 & 1 & 73 & & & 12 & & 80 & & & 2 & 13 & & 100 \\
\hline ELAEOCARPACEAE & Vallea stipularis L. f. * & & & & & & & & 9 & 4 & 87 & & & 11 & 3 & 93 & & & 2 & 9 & 73 & & & & & & \\
\hline ESCALLONIACEAE & Escallonia resinosa (Ruiz \& Pav.) Pers* & 10 & 5 & & & 100 & & & & & & & 15 & & & 100 & 15 & & & & 100 & 3 & 12 & & & & 100 \\
\hline \multirow[t]{2}{*}{ EUPHORBIACEAE } & Acalypha aronioides Pax \& Hoffmann* & & & & & & & & & 4 & 27 & & & & 5 & 33 & & & & 5 & 33 & & & & & & \\
\hline & Sebastiana $s p *$ & & & & & & & & & & & & & & & & & & & & & & & & & 3 & 20 \\
\hline \multirow[t]{14}{*}{ FABACEAE } & Caesalpinia spinosa (Molina) Kuntze * & & & & & & & & & & & & & & 7 & 47 & & & & 4 & 27 & & & & & & \\
\hline & Crotalaria incana L. * & & & & & & & & & & & & & & & & & & & 3 & 20 & & & & & 6 & 40 \\
\hline & Dalea sp * & & & & & & & & & & & & & & & & & & & 4 & 27 & & & & 1 & 8 & 60 \\
\hline & Desmodium $s p *$ & & & & & & & & & & & & & & & & & & & 3 & 20 & & & & & 3 & 20 \\
\hline & Erythrina falcata Bentham * & & & & 8 & 53 & & & & 8 & 53 & & & & 6 & 40 & & & & & & & & & & & \\
\hline & Inga $s p$ & & & & & & & & & & & & & & 1 & 7 & & & & & & & & & & & \\
\hline & Medicago sp & & & 5 & 6 & 73 & & & 9 & 6 & 100 & & & 11 & 4 & 100 & & & 12 & 3 & 100 & & & & 3 & 8 & 73 \\
\hline & Melilotus sp & & & & & & & & 9 & 6 & 100 & & & 8 & 7 & 100 & & & 14 & & 93 & & & & 8 & 5 & 87 \\
\hline & Mimosa sp & & & & & & & & & & & & & & 2 & 13 & & & & & & & & & & 2 & 13 \\
\hline & Otholobium pubescens (Poiret) Grimes* & & & & 9 & 60 & & & & 9 & 60 & & & & 8 & 53 & & & & 7 & 47 & & & & & 5 & 33 \\
\hline & Spartium junceum L. & & & & 7 & 47 & & & & 7 & 47 & & & & 6 & 40 & & & & 5 & 33 & & & & & 5 & 33 \\
\hline & Telima monspessulanus L. & & & 10 & & 67 & & & 9 & 1 & 67 & & & 7 & 2 & 60 & & & 11 & & 73 & & & & 8 & 1 & 60 \\
\hline & Trifolium sp & & 3 & 10 & 2 & 100 & & 15 & & & 100 & & 15 & & & 100 & & 14 & 1 & & 100 & & & 9 & 6 & & 100 \\
\hline & Vicia $s p$ & & & & & & & & & & & & & & 3 & 20 & & & & 4 & 27 & & & & & & \\
\hline JUGLANDACEAE & Juglans neotropica Diels * & & & & 7 & 47 & & & & & & & & & & & & & & & & & & & & 5 & 33 \\
\hline \multirow[t]{4}{*}{ LAMIACEAE } & Leonotis nepetifolia (L.)R. Br. & & & & 8 & 53 & & & 2 & 5 & 47 & & & 3 & 5 & 53 & & & 1 & 10 & 73 & & & & 3 & 6 & 60 \\
\hline & Lepechinia floribunda (Benth.) Epling * & & & & & & & & & & & & & & & & & & & & & & & & & 6 & 40 \\
\hline & Minthostachys $s p$ * & & & & & & & & & & & & & & & & & & & & & & & & & 6 & 40 \\
\hline & $\begin{array}{l}\text { Rosmarinus officinalis L. } \\
\text { Stachys sp* }\end{array}$ & & & & 11 & 73 & & & & 9 & 60 & & & & 9 & 60 & & & & 3 & 20 & & & & & 11 & 73 \\
\hline
\end{tabular}


Tabla 1. Clases de frecuencia y frecuencia de ocurrencia de los 113 morfotipos polínicos determinados en los 5 periodos de producción en la localidad de Huarán para la temporada apícola 2012-2013.

\begin{tabular}{|c|c|c|c|c|c|c|c|c|c|c|c|c|c|c|c|c|c|c|c|c|c|c|c|c|c|c|c|}
\hline \multirow{2}{*}{ FAMILIA } & \multirow{2}{*}{ MORFOTIPO POLÍNICO } & \multicolumn{5}{|c|}{ Periodo I } & \multicolumn{5}{|c|}{ Periodo II } & \multicolumn{5}{|c|}{ Periodo III } & \multicolumn{5}{|c|}{ Periodo IV } & \multicolumn{6}{|c|}{ Periodo V } \\
\hline & & $\mathrm{S}$ & I & $\mathrm{T}$ & $(+)$ & FO & $\mathrm{S}$ & I & $\mathrm{T}$ & $(+)$ & FO & $\mathrm{S}$ & I & $\mathrm{T}$ & $(+)$ & FO & $\mathrm{S}$ & I & $\mathrm{T}$ & $(+)$ & FO & $\mathrm{D}$ & $\mathrm{S}$ & $\mathrm{I}$ & $\mathrm{T}$ & $(+)$ & FO \\
\hline LAURACEAE & Persea americana Mill. * & & & & 6 & 40 & & & & & & & & & & & & & & 6 & 40 & & & & & 6 & 40 \\
\hline LORANTHACEAE & Gaiadendron punctatum (R. \& P.) Don* & & & & 9 & 60 & & & 15 & & 100 & & & 15 & & 100 & & & 13 & 2 & 100 & & & & 3 & 10 & 87 \\
\hline MALVACEAE & Malvaceae & & & & & & & & & 4 & 27 & & & & & & & & & 3 & 20 & & & & & 3 & 20 \\
\hline MYRICACEAE & Morella pubescens (H. \& B.) Wilbur * & & & & & & & & & & & & & 4 & 11 & 100 & & & 1 & 9 & 67 & & & & & & \\
\hline \multirow{2}{*}{ MYRTACEAE } & Eucalyptus $s p$ & 11 & 4 & & & 100 & 15 & & & & 100 & 15 & & & & 100 & 8 & 7 & & & 100 & & & 15 & & & 100 \\
\hline & Luma chequen (Molina) A. Gray & & & & & & & & & & & & & 4 & 3 & 47 & & & 3 & 3 & 40 & & & & & & \\
\hline ONAGRACEAE & Oenothera $s p *$ & & & & & & & & & 5 & 33 & & & & 6 & 40 & & & & 6 & 40 & & & & & 5 & 33 \\
\hline OXALIDACEAE & Oxalis $s p^{*}$ & & & & & & & & 4 & 5 & 60 & & & 4 & 6 & 67 & & & 5 & 5 & 67 & & & & 3 & 4 & 47 \\
\hline PASSIFLORACEAE & Passiflora sp * & & & & 6 & 40 & & & & 6 & 40 & & & & 7 & 47 & & & & 8 & 53 & & & & & 6 & 40 \\
\hline PHRYMACEAE & Mimulus glabratus Kunth * & & & & & & & & & 3 & 20 & & & & 2 & 13 & & & & 3 & 20 & & & & & & \\
\hline \multirow[t]{2}{*}{ PLANTAGINACEAE } & Plantago $s p^{*}$ & & & & & & & & & & & & & & 5 & 33 & & & & 4 & 27 & & & & & 3 & 20 \\
\hline & Veronica sp & & & & 7 & 47 & & & 1 & 6 & 47 & & & 4 & 2 & 40 & & & 1 & 8 & 60 & & & & 1 & 5 & 40 \\
\hline \multirow{2}{*}{ POACEAE } & Poaceae & & & & & & & & & & & & & & & & & & & 2 & 13 & & & & & 2 & 13 \\
\hline & Zea mays L. & & & & & & & & & & & & & & 2 & 13 & & & & 3 & 20 & & & & & & \\
\hline POLYGONACEAE & Rumex sp* & & & & & & & & & & & & & & 4 & 27 & & & & 3 & 20 & & & & & 4 & 27 \\
\hline RANUNCULACEAE & Thalictrum $s p^{*}$ & & & & & & & & & & & & & & & & & & & 3 & 20 & & & & & 3 & 20 \\
\hline RHAMNACEAE & Colletia spinosissima J.F. Gmel. * & & & & 5 & 33 & & & 1 & 6 & 47 & & & & 4 & 27 & & & & 3 & 20 & & & & & & \\
\hline \multirow[t]{9}{*}{ ROSACEAE } & Cydonia oblonga Mill. & & & & & & & & & 4 & 27 & & & & & & & & & & & & & & & & \\
\hline & Hesperomeles $s p *$ & & & & & & & & & 6 & 40 & & & & 5 & 33 & & & & & & & & & & & \\
\hline & Kageneckia lanceolata Ruiz \& Pav.* & & & & & & & & & & & & & & 3 & 20 & & & & & & & & & & & \\
\hline & Malus domestica Borkh. & & & & & & & & & 9 & 60 & & & & 6 & 40 & & & & 9 & 60 & & & & & & \\
\hline & Mespilus germanica L. & & & & 7 & 47 & & & & 9 & 60 & & & & 8 & 53 & & & & & & & & & & & \\
\hline & Prunus serotina Ehrh. & 4 & 11 & & & 100 & 15 & & & & 100 & & 15 & & & 100 & & 15 & & & 100 & & & 11 & 4 & & 100 \\
\hline & Prunus $s p$ & 15 & & & & 100 & & 15 & & & 100 & & 13 & 2 & & 100 & & 3 & 9 & & 80 & & & 10 & 4 & & 93 \\
\hline & Pyrus communis L. & & & & & & & & & 8 & 53 & & & & 9 & 60 & & & & & & & & & & & \\
\hline & Rubus sp & & & & & & & & 1 & 5 & 40 & & & & 5 & 33 & & & & 7 & 47 & & & & & 3 & 20 \\
\hline \multirow[t]{2}{*}{ RUTACEAE } & Citrus sp & & & & & & & & & & & & & & & & & & & & & & & & & 3 & 20 \\
\hline & Ruta $s p$ & & & & 3 & 20 & & & & 3 & 20 & & & & 3 & 20 & & & & 3 & 20 & & & & & 3 & 20 \\
\hline \multirow[t]{2}{*}{ SALICACEAE } & Pineda incana Ruiz \& Pav. * & & & & & & & & & & & & & & 4 & 27 & & & 4 & 5 & 60 & & & & & & \\
\hline & Salix $s p *$ & & & & 5 & 33 & & & & 8 & 53 & & & & 8 & 53 & & & & & & & & & & & \\
\hline SAPINDACEAE & Serjania squarrosa Radlk. * & & & & & & & & & & & & & & 3 & 20 & & & & 4 & 27 & & & & & 4 & 27 \\
\hline \multirow[t]{3}{*}{ SOLANACEAE } & Brugmansia sp * & & & & & & & & & 2 & 13 & & & & & & & & & 3 & 20 & & & & & & \\
\hline & Nicotiana tomentosa Ruiz \& Pav.* & & & & 7 & 47 & & & 1 & 7 & 53 & & & 1 & 7 & 53 & & & 2 & 7 & 60 & & & & 5 & 2 & 47 \\
\hline & Solanum $s p^{*}$ & & & & & & & & & & & & & & 3 & 20 & & & & 4 & 27 & & & & & 2 & 13 \\
\hline TROPAEOLACEAE & Tropaeolum $s p *$ & & & & & & & & & & & & & & 5 & 33 & & & & 7 & 47 & & & & & 8 & 53 \\
\hline URTICACEAE & Urticaceae & & & & & & & & & & & & & & 2 & 13 & & & & 2 & 13 & & & & & & \\
\hline VERBENACEAE & Aegiphila mortonii Moldenke* & & & & & & & & & & & & & & & & & & & & & & & & & 2 & 13 \\
\hline
\end{tabular}


Tabla 1. Clases de frecuencia y frecuencia de ocurrencia de los 113 morfotipos polínicos determinados en los 5 periodos de producción en la localidad de Huarán para la temporada apícola 2012-2013.

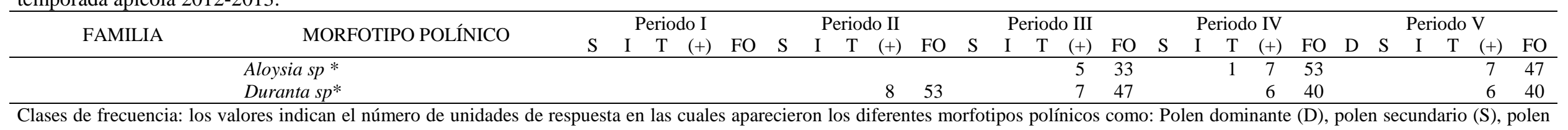
de menor importancia (I), polen en traza (T), polen presente $(+)$. FO: frecuencia de ocurrencia (en porcentaje). * Especies nativas.

Tabla 2. Valores singulares, porcentaje de inercia, porcentaje acumulado y valores Chi-Cuadrado para los 3 primeros ejes en el Análisis de Correspondencia.

\begin{tabular}{rrrrrr}
\hline & Autovalor & Inercia & Chi-Cuadrado & (\%) de inercia & (\%) acumulado \\
\hline 1 & 0.45 & 0.20 & 1500.87 & 48.82 & 48.82 \\
2 & 0.39 & 0.15 & 1160.75 & 37.75 & 86.57 \\
3 & 0.19 & 0.04 & 274.25 & 8.92 & 95.49 \\
\hline
\end{tabular}

1 Sociedad Botánica del Cusco, Facultad de Ciencias, Universidad Nacional de San Antonio Abad del Cusco, Ciudad Universitaria, Av. De la Cultura s/n, Cusco, Perú. rivas_84_zu@hotmail.com. 